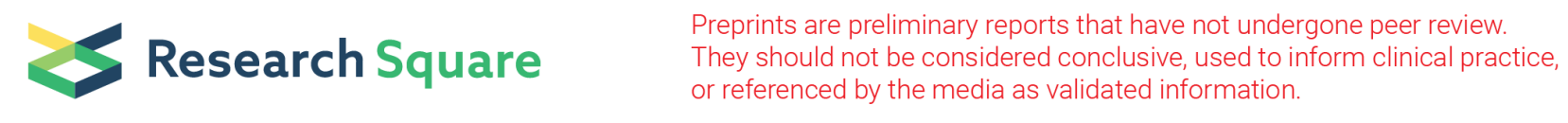

\title{
Effect of volatile and intravenous anesthesia on long-term survival of cancer surgery patients: a systematic review and meta-analysis
}

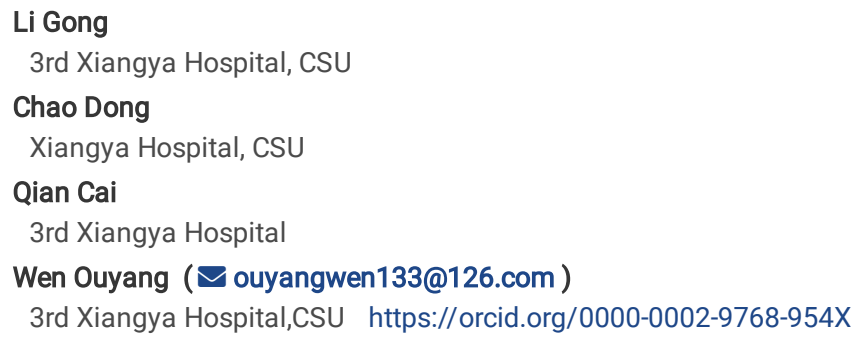

\section{Research article}

Keywords: anesthesia, intravenous, inhalational, neoplasm recurrence, overall survival

Posted Date: January 9th, 2020

DOI: https://doi.org/10.21203/rs.2.116/v2

License: @) (1) This work is licensed under a Creative Commons Attribution 4.0 International License. Read Full License 


\section{Abstract}

Background The impact of volatile anesthesia (INHA) and total intravenous anaesthesia (TIVA) on the long-term survival of patients after oncology surgery is a subject of controversy. The purpose of this study was to make overall evaluation of the association between these two anesthetic techniques and long-term prognosis of oncology patients after surgery.

Methods Databases were searched according to the PRISMA guidelines up to September 30, 2018. Hazard ratios (HRs) with its $95 \%$ confidence intervals (Cls) were calculated after multivariable analyses and propensity score (PS) adjustments. Eight retrospective cohort articles reporting data on overall survival (OS) and recurrence-free survival (RFS) were included. An inverse variance random effects meta-analysis was conducted. The Newcastle Scale was used to assess methodological quality and bias.

Results In total, about 18922 cancer patients observed were included in the meta-analysis, of which 10433 cases were available for analysis in INHA and 8489 in TIVA group. Compared to TIVA, INHA showed a shorter OS (HR=1.27, 95\% Cl 1.069 to $1.516, p=0.007)$, with a medium heterogeneity (Q-test $p=0.003$, Isquared $=67.6 \%$ ). However, no significant differences were identified between INHA and TIVA group (HR=1.10, $95 \% \mathrm{Cl} 0.729$ to $1.659, \mathrm{p}=0.651$ ) concerning RFS albeit from a limited data pool. When a subgroup analysis was performed by race, the association was more likely to be observed in the Asian studies $(\mathrm{HR}=1.46,95 \% \mathrm{Cl} 1.19-1.8, \mathrm{p}=0.00)$, with a much lower heterogeneity (Q-test $\mathrm{p}=0.148$, l-squared=44\%). When comparison was done only in breast cancer patients, no significant differences were found for OS (HR=1.625, 95\% CI 0.273-9.67, p=0.594) between INHA and TIVA.

Conclusion TIVA for cancer surgery might be associated with better OS compared to INHA. The effect of INHA and TIVA on OS and RFS in the perioperative setting remains uncertain, cancer-specific, and has low-level evidence at present. Randomized controlled trials are required in future work. Registry number The review protocol was registered with PROSPERO (Registration NO.CRD42018109341).

\section{Background}

The perioperative period is a critical stage for malignant cells seeding, invading, and metastasis. Growing commentary suggests that numerous perioperative factors can facilitate the cancer progression, such as surgical stress, volatile anesthetics, opioids, blood transfusion, postoperative pain and so on [1-3]. Both intravenous and volatile inhalational anesthetics are two main classes of drugs administrated at a window period of potentially high vulnerability in terms of the dissemination and establishment of metastasis [4]. And many studies have investigated the differences between the two agents in systemic immune function, hemodynamics, complications, short and long-term outcomes after operation[5-7]. It is shown that inhalation agents appear to have the proinflammatory effect and to alter the immune processes, thereby on the one hand promoting the metastasis of lung and breast cancer in mice and humans [8-9]. But on the other hand, propofol has been demonstrated to exert predominately anticancer properties through multiple mechanisms, such as through suppression of the vascular proliferation and regeneration associated with surgery, anti-inflammatory and antioxidative activities and so on [10-13]. These mechanisms all lead to the notion that the administration of inhalation agent in surgery may facilitate cancer prognosis while propofol has a beneficial effect.

Recently, some clinical studies in various surgical disciplines have sought to determine whether propofol-based total intravenous anesthesia (TIVA) improves survival after oncologic surgery compared with inhalational anesthesia (INHA) [14-21]. But findings from these studies assessing the use of these two anesthetic techniques and long-term survival in cancer patients are inconsistent. Until now, only one systematic review concerning the impact of INHA and TIVA on the outcomes after cancer surgery was published in 2017 [22]. As the evidence is based on a limited number of published papers, there is lack of data to make any clear conclusions. In addition, since this systematic review was published, five more papers reporting conflict results have been found[17-21]. To date, there has been not quantitative meta-analysis dedicated to the influence of INHA and TIVA on long-term survival after cancer surgery. Hence, we conducted a comprehensive literature review and meta-analysis of cohort studies to compare the effects of INHA and TIVA on long-term survival in patients after cancer operation. The results of this study can add to the evidence for better choice of the anesthetic technique to maximize long-term outcomes.

\section{Methods}

\section{Protocol and registration}

This systematic review and meta-analysis were complied with the Preferred Reporting Items.

These items are especially made for Systematic Reviews and Meta-Analyses (PRISMA) guidelines for system reviews [23]. And a review protocol was registered with PROSPERO (Registration NO.CRD42018109341).

\section{Search strategy and eligibility criteria}

Relevant publications were searched in Pubmed, the Cochrane Central Register of Controlled Trials, Scopus, and EMBASE databases up to September 30 , 2018. The search terms included "intravenous Anesthesia ", "inhalational Anesthesia", "cancer surgery" and "survival". In addition, we used the specific name of anesthetics to recognize additional studies, such as "propofol", "sevoflurane". All the articles were separately screened and identified by two review authors ( $L G$ and $C D$ ) to ensure sensitivity of the search strategy. Only English language and the full-text papers were eligible. And no time restrictions were applied to the research. Based on a full review of the retrieved articles, study eligibility was assessed according to the PICOS (Populations, Interventions, Comparisons, Outcomes, and Study designs) approach.

1). Populations $(P)$ : The sample size of the included studies is more than 50 . The population was adult patients undergoing oncologic surgery.

2). Intervention (I) and Comparison (C): compared the long-term outcomes between patients using INHA and TIVA. 
3). Outcomes (0): provided important information, including hazard ratios (HRs) and 95\% confidence intervals (Cls) for overall survival (OS) or recurrence-free survival (RFS). All the non-adjusted, adjusted by multivariate Cox regression or propensity score (PS) data were obtained from each study. Some more data including the size of baseline samples, the total number of cases, the overall survival rate, and the confounders adjusted in final models, were available. The time of follow-up is at least 3 years.

4). Study design (S): designed as a case-control or cohort study or clinical trial.

Studies were excluded, if they were a protocol, meta-analysis, literature review, letter to the editor or abstract without full text. Studies with insufficient available data to estimate a HR with $95 \%$ Cls were removed. Studies with an English abstract but not with an English full text were neither considered.

\section{Data Extraction and quality assessment}

All data and items helpful to assess the validity of the studies were independently extracted by two of the reviewers. Any different opinions between the reviewers were solved through repeated discussion, to finally reach a consensus. To remove any potential systematic biases, other two authors did a comparable study by independently scanning all the eligible publications. After this process we agreed on all the variables assessed.

The following variables were extracted from each paper where the following information was available: authors' surname, publication year, country or district, sample size before and after propensity score matching of both groups, design type, anesthetic agents, outcomes, adverse effect, median follow-up, survival rate, type of cancer surgery, and hazard ratios (HR) with 95\% confidence intervals (CI). An adverse effect study was defined as being the worse longterm survival after surgery in INHA group compared with TIVA. If different groups were divided into by volatile anesthetics as shown in Kim's study [17], all the relevant intervention groups of the study were combined as a single treatment group. Consequently, both the sample sizes and the quantity of people with intervention events were respectively summed across groups.

The time-to-event data was retrieved from Kaplan-Meier curves, log-rank statistics or multivariate Cox regression analysis results of the individual trials. It was summarized in terms of the HR in logarithm scale as a function of its variance. Non-adjusted (by univariate analysis), multivariable-adjusted, multivariable and PS-adjusted estimates (HR and 95\% Cl) for OS and RFS in all the studies were separately extracted.

When the outcome data for OS or RFS was unavailable, the Excel spread sheet freely provided by Tierney et al. and the software Engauge Digitizer, version 4.1 (downloaded from http://sourceforge.net), were used to regenerate data points from the Kaplan-Meier curves. Authors of the selected published articles were contacted by email whenever we found that data indispensable for the meta-analysis was not explicitly provided. If the HR and $95 \% \mathrm{Cl}$ of TIVA to INHA for OS and RFS were reported, it would be converted into the HR and $95 \% \mathrm{Cl}$ of INHA to TIVA.

Quality of the included studies was separately assessed by the Newcastle-Ottawa Score (NOS). Low quality was defined as the score less than 8.

\section{Statistical Analysis}

Both Cochran chi-square Q statistics and I-square statistics were used to assess the heterogeneity of the included studies. Heterogeneity was considered as either a $P$ value $\leq 0.10$ [26]. It also determined the appropriate use of random-effects (DerSimonian and Laird method) model. And when the l-square statistic was used to measure the consistency of effects across studies, $20-50 \%, 50-75 \%$, and $>75 \%$ were considered low, moderate, and high heterogeneity, respectively. Potential predictors of heterogeneity were further explored by sub-group analysis.

Sensitivity analysis was explored through the different types of estimate (unadjusted, multivariable analysis-adjusted or both multivariable and PS-adjusted). Meta-regression analyses were explored the effect of study quality on the results.

The potential publication bias of this meta-analysis was judged visually in a funnel plot of In [HR] against its standard error (SE In [HR]), and the degree of asymmetry was examined by the Egger's and Begg's test if the included study number is not less than 8 . We considered a significant publication bias if a $p$ value $<0.1$. We further used the trim and fill computation to estimate the effect of publication bias on the explanation of results.

Subgroup analyses were respectively performed on high-quality versus low-quality studies (NOS variable), and Non-Asian versus Asian studies. The pooled HR estimates derived from the two separate analyses were compared with a test of interaction.

Time-to-event outcomes were appropriately analysed and compared graphically using the hazard ratio of non-adjusted effect sizes, adjusted by multivariable analysis, or both multivariable and PS-adjusted. The log HR and its variance were pooled using an inverse variance weighted average, and the results were presented as an $\mathrm{HR}$ and $95 \% \mathrm{Cl}$. All of the statistical analyses in this meta-analysis were conducted with Stata/SE version 14 (Stata Corporation, College Station, TX 77845), using the commands metan, metareg and metabias.

\section{Results}

\section{Literature Search}

As shown in Figure 1, a literature search flowchart is presented in detail. In brief, from 1537 citations researched, 12 were retained based on the title and abstract. Of these 12 , only 8 papers qualified for inclusion and data retrieval in this meta-analysis.

\section{Basic characteristics}


We investigated 8 eligible articles for this meta-analysis [14-21], consisting of 18922 cases in which 10433 cases were found in INHA and 8489 in TIVA group (Table 1). And all the studies were retrospective cohort studies published from 2014 to 2018. All were written in English. And six of the eight articles were carried out in Asian.

The detailed characteristics of the included studies are summarized in Table 1. From the 8 articles of the systemic review, a total of 10 studies were analysed given that the reported different effect size by cancer type (Enlund(1), (2) and (3) 2014) were considered as different studies. One study compared the effects of INHA versus TIVA on long-term survival in more than one cancer type [Wigmore 2016]. Five studies compared the effect of two groups in breast cancer [Enlund(3) 2014, Lee 2016, and Kim 2017], and two did in colon cancer [Enlund(1) 2014, and Wu 2018].

TIVA in all the studies is propofol-based. Different kinds of volatile anesthetics were reported in INHA group in these studies, but only one kind was administrated in each study. In addition, various opioids were used in both groups of these studies.

The HRs for OS adjusted by multivariable analysis and PS, were reported or calculated in eight studies. But only three studies reported the HR of effect sizes adjusted by multivariable analysis and PS for RFS.

All studies included adjusted for several of the following variables in their multivariate analyses: age, body mass index, transfusion, time of surgery, use of regional analgesia, comorbidities, preoperative therapy, pathologic stage or grade of cancer, radiotherapy, presence of postoperative metastasis and so on.

\section{Association between these two anesthetic techniques and long-term survival}

The data of each outcome are summarized in Table 2. All studies reporting both multivariate and PS adjusted data were analysed and combined. Studies reporting multivariate and PS adjusted data were included as follows (Figure 2):

OS: 8 studies showed a pooled HR of $1.273(95 \% \mathrm{Cl}=1.069-1.516, \mathrm{p}=0.007)$, with moderate heterogeneity $(\mathrm{Q}$-test $\mathrm{p}=0.003, \mathrm{I}=67.6 \%)$. Heterogeneity was obviously reduced ( $Q$-test $p=0.148, \mathrm{I}^{2}=44 \%$ ) when only the Asian studies were included (HR 1.46, 95\%Cl=1.19-1.8, $\left.\mathrm{p}=0.00\right)$.

And 3 studies showed a pooled HR of $1.95(95 \% \mathrm{Cl}=0.414,9.184, \mathrm{p}=0.398)$ concerning postoperative OS of breast cancer, with moderate heterogeneity (Q-test $\left.p=0.060, l^{2}=64.5 \%\right)$.

RFS: 3 adjusted studies revealed a pooled HR of $1.1(95 \% \mathrm{Cl}=0.729-1.659, \mathrm{p}=0.651)$, with moderate heterogeneity $\left(\mathrm{Q}\right.$-test $\left.\mathrm{p}=0.276, \mathrm{I}^{2}=59.3 \%\right)$.

3 adjusted studies showed a pooled HR of $1.284(95 \% \mathrm{Cl}=0.592,2.785, \mathrm{p}=0.527)$ concerning postoperative DFS of breast cancer, with low heterogeneity ( $\mathrm{Q}$-test $\left.p=0.909,\left.\right|^{2}=0 \%\right)$.

No significant differences exist between INHA and TIVA group ( $\mathrm{HR}=1.625,95 \% \mathrm{Cl} 0.273$ to $9.67, \mathrm{p}=0.594)$ concerning postoperative 0 S in breast cancer.

The mean of NOS score was 7 for studies reporting OS, 7.7 for those reporting RFS. In the subgroup analysis, no significant differences were shown in the OS of Non-Asian vs. Asian studies (HR 1.11, 95\% Cl 0.831-1.487 vs. HR 1.46, 95\% Cl 1.19-1.8, p = 0.614) and high quality studies vs. low quality studies (HR 1.51, $95 \% \mathrm{Cl} 1.28-1.78$ vs. HR 1.13 vs. $95 \% \mathrm{Cl} 0.868-1.463 ; \mathrm{p}=0.55)$ concerning OSA graphic comparison displayed a reduced effect size in both PS and multivariate adjusted data both for OS and RFS, compared to unadjusted or multivariate adjusted data(Figure 3 ).

\section{Publication bias}

The quantity of studies was consistent for OS adjusted by multivariable and PS; and thus we made a funnel plot (Figure 3). Either graphical inspection for funnel plots or quantitative evaluation from the Begg's test $(p=0.711)$ and Egger's test $(p=0.449)$ indicated the presence of publication bias in the overall survival. But the trim-and fill method was further performed to show that the publication bias did not impact the estimates.

\section{Discussion}

This systemic review represents a thorough assessment of the current literature in the realm of anesthetic techniques and long-term prognosis of oncology patients after reductive operation. Based on the eight observational articles published from 2014 to 2018 , our review suggests that INHA is associated with reduced OS compared to TIVA. But INHA is shown to have no consistent relation with the worse RFS, and similar effects were found in specific types of cancer (e.g. breast and colon cancer), albeit from a sparse data pool. And in other two meta-analysis literature[24-25], HRs adjusted by multivariate analysis were harvested and combined. But our meta-analysis was completely conducted on data adjusted by two different statistical risk adjustment methods including multivariate analysis and PS, as recommended by the current validated methodological guidelines [26-28]. We also found a reduced effect size in both PS and multivariate adjusted data both for OS and RFS, compared to unadjusted or multivariate adjusted.

Establishing a causal relationship between the two anesthetic techniques and cancer survival is challenging because some perioperative risk factors are also associated with cancer outcome, such as transfusion, epidural anesthesia[2, 29]. Thus, there may be great differences in the observed covariates, which can produce biased estimates of intervention effects. Although unknown confounders cannot be corrected, multivariate analysis allows consistent adjustment for multiple covariates in logistic regression models[30-31].But it may still be inadequate to eliminate this bias. The adjusted data by both multivariate and PS was proven to be valid through a graphical comparison in figure 3, even though only the unadjusted data was analysed in most past meta-analysis. PS matching allows for the management of numerous covariates at the same time and thus help to process the data and design regression analyses, although unmeasured variables being imbalanced between the subjects still exist[32-33]. In our research, twelve studies included in the review compared INHA and TIVA by PS matching and multivariate analysis. And INHA showed an unfavourable OS. Growing evidence from previous experimental investigations supports a

Page $4 / 12$ 
role for volatile agents in adversely affecting the long-term outcome. For example, it is reported that inhaled anesthesia may raise the risk of cancer cells evading immune surveillance and control[34]. It can extract on lymphocytes apoptosis inducton, natural killer (NK) cytotoxicity inhibition and cytokines release alteration by NK cells in response to malignant cells, ultimately contributing to the development of migration and recurrence postoperatively[35]. However, as the most popular and common choice in TIVA, propofol has been considered to have predominately anticancer effects[10-13]. It can boost immune functions of the body and thus inhibit the cancer spread and metastasis[10-13]. Especially, propofol infusion anaesthesia has been found to modulate the Th1/ 2 balance by increasing the IFN-y/IL-4 ratio in vitro[34]. In addition, propofol has apoptotic effects on tumor cells and changes the pro-angiogenic state related with surgical stress[10-11]. When stratified by race, the conclusion is identical in Asian people and the heterogeneity can be obviously improved. This was also indicative of different races as one of the main sources of heterogeneity.

But contrary to OS, no significant difference was found between INHA and TIVA with regard to RFS. Besides, the effects of INHA are comparable with those of TIVA regarding postoperative OS and RFS in breast cancer patients ( $\mathrm{HR}=1.95,95 \% \mathrm{Cl} 0.414$ to $9.184, \mathrm{p}=0.00$, and $\mathrm{HR}=1.284,95 \% \mathrm{Cl} 0.592$ to 2.785 , $\mathrm{p}=0.527$ ). How to explain the inconsistency? As noted above, the uses of epidural analgesia, opioids, transfusion and so on, have been reported as key factors influencing long-term surviving of cancer patients[1-4, 29]. But not all the studies took these factors in account. For instance, epidural analgesia was not used or considered in some studies[16, 20], but was in other studies[14-15,17-19,21]. In addition, differences in surgical techniques and cancer types, varying patient populations, and different anesthetics exposure time all hamper to obtain firm conclusions[37-38]. And when only the breast cancer considered, TIVA was not associated with an improvement both in RFS and OS compared to INHA. Again to note, different cancer patients with various pathological pattern cancer stage, metastatic potential and biology were included in this finding. Some cancers may progress very rapidly. And advanced stages can also eventually give rise to clinically manifested relapse, thus it is related closely to the prognosis of the patients[39]. Besides, inadequate data and limited number of studies reporting RFS (only five studies from three articles) after correction, may also account for the inconsistent results, limiting the ability to explore the association between the two anesthetic techniques and long-term survival. In summary, it confirms the hot disputation over an optimal choice of anesthetic technique for cancer operation and suggests the necessary for further well-designed randomized controlled trials.

Some limitations in this study should be stated. First, this meta-analysis was subject to the retrospective nature of the included articles while the gold standard of scientific evidence is the prospective randomized controlled trial [40]. And two prospective clinical trials (NCT02660411, NCT03447691) currently registered to compare between TIVA and INHA on long-term outcomes of patients undergoing cancer operation. Second, it is known that inadequate study design, improper inclusive criteria, or wrong statistical method; all may directly influence the results [41]. In addition, some demographic and surgical variables such as the patients' age, sex, cancer site, need for extensive operation, quantity of major complications after surgery, regular medication may also hamper to draw firm conclusions. Although all studies included adjusted for several variables in their multivariate analyses, important factors such as presence of postoperative metastasis was only adjusted in one study[21]. Especially, their multivariate analyses were not adjusted for regular medication such as betablocker drugs, while some drugs may affect the cancer survival distinctly [42]. Third, as the funnel plot showed some asymmetry, the presence of possible

publication bias might have led to an overestimate of the association between the anesthetic technique and the survival rate. And some small researches with null results were not likely to be published. Moreover, the full-text of studies which were only published in English were harvested for analyse. And this may decrease the precision of the combined estimates of the intervention effects. This limitation, however, can also be found in most recently published metaanalysis and systemic reviews.

In conclusion, TIVA may be the preferred anaesthetic for cancer resection surgery compared to INHA. Nevertheless, to make the conclusion more straightforward, large prospective randomized controlled trials are required to further investigate the potential beneficial effect of TIVA compared to INHA in future.

\section{List Of Abbreviations}

INHA inhalational anesthesia, TIVA total intravenous anaesthesia, PS propensity score,

HR Hazard ratios, Cl confidence interval, OS overall survival, RFS recurrence-free survival

\section{Declarations}

Ethics approval and consent to participate: Not applicable.

Consent for publication: Not applicable.

Availability of data and material: The datasets used and analysed during the current study are available from the corresponding author on reasonable request.

Competing interests: The authors declare that they have no competing interests.

Funding: This study is supported by National Key R\&D Program of China (2018YFC2001800) and Hunan Science and Technology Innovation Program Project (No. 2018SK2094).

Authors' contributions: LG, CD and WO searched and collected the data. LG and WO analysed the data and wrote the paper. WO, CD and QC reviewed and edited the manuscript. All authors read and approved the manuscript

Acknowledgments: None.

\section{References}


1. Tsuchiya Y, Sawada S, Yoshioka I, Ohashi Y, Matsuo M, Harimaya Y, et al. Increased surgical stress promotes tumor metastasis. Surgery. 2003;133(5):547-55.

2. Acheson A G, Brookes M J, Spahn D R. Effects of allogeneic red blood cell transfusions on clinical outcomes in patients undergoing colorectal cancer surgery: a systematic review and meta-analysis. Annals of surgery. 2012;256(2):235-244.

3. Tavare A N, Perry N J S, Benzonana L L, Takata M, Ma D. Cancer recurrence after surgery: direct and indirect effects of anesthetic agents. International journal of cancer. 2012;130(6):1237-1250.

4. Fodale V, D’Arrigo M G, Triolo S, Mondello S, La Torre D. Anesthetic techniques and cancer recurrence after surgery. ScientificWorldJournal. 2014;2014:328513.

5. Mirabella L, Bellanti F, Tullo L, Serviddio G, Cinnella G, Dambrosio M. Effect of sevoflurane and propofol anaesthesia on mitochondria and postoperative hepatocellular injury in steatotic patients: preliminary data: 9AP5-7. European Journal of Anaesthesiology. 2013;30:153-153.

6. Ren XF, Li WZ, Meng FY, Lin CF. Differential effects of propofol and isoflurane on the activation of T-helper cells in lung cancer patients. Anaesthesia. 2010;65:478-482.

7. Herling SF, Dreijer B, Lam GW, Thomsen T, Møller AM. Total intravenous anaesthesia versus inhalational anaesthesia for adults undergoing transabdominal robotic assisted laparoscopic surgery. Cochrane Database of Systematic Reviews. 2017;(4).

8. Benzonana LL, Perry NJ, Watts HR, Yang B, Perry IA, Coombes C, et al. Isoflurane, a commonly used volatile anesthetic, enhances renal cancer growth and malignant potential via the hypoxia-inducible factor cellular signaling pathway in vitro. Anesthesiology. 2013;119:593-605.

9. Loop T, Dovi-Akue D, Frick M, Roesslein M, Egger L, Humar M, et al. Volatile anesthetics induce caspase-dependent, mitochondria-mediated apoptosis in human T lymphocytes in vitro. Anesthesiology. 2005;102:1147-57

10. Zhang D, Zhou XH, Zhang J, Zhou YX, Ying J, Wu GQ, et al. Propofol promotes cell apoptosis via inhibiting HOTAIR mediated mTOR pathway in cervical cancer. Biochemical and biophysical research communications. 2015;468(4):561-567.

11. Song J, Shen Y, Zhang J, Lian Q. Mini profile of potential anticancer properties of propofol. PLoS One 2014;9:e114440.

12. Yang C, Gao J, Yan N, Wu B, Ren Y, Li H, Liang J. Propofol inhibits the growth and survival of gastric cancer cells in vitro through the upregulation of ING3. Oncol Rep. 2017;37:587-593.

13. Kushida A, Inada T, Shingu K. Enhancement of antitumor immunity after propofol treatment in mice. Immunopharmacol Immunotoxicol. 2007;29:477-86.

14. Enlund M, Berglund A, Andreasson K, Cicek C, Enlund A, Bergkvist L. The choice of anaesthetic-sevoflurane or propofol-and outcome from cancer surgery: a retrospective analysis. Upsala journal of medical sciences. 2014;119(3):251-261.

15. Wigmore T J, Mohammed K, Jhanji S. Long-term Survival for Patients Undergoing Volatile versus IV Anesthesia for Cancer SurgeryA Retrospective Analysis. Anesthesiology. 2016;124(1):69-79.

16. Lee JH, Kang SH, Kim Y, Kim HA, Kim BS. Effects of propofol-based total intravenous anesthesia on recurrence and overall survival in patients after modified radical mastectomy: a retrospective study. Korean journal of anesthesiology. 2016;69(2):126-132.

17. Kim MH, Kim DW, Kim JH, Lee KY, Park S, Yoo YC. Does the type of anesthesia really affect the recurrence-free survival after breast cancer surgery? Oncotarget. 2017;8(52):90477.

18. Jun IJ, Jo JY, Kim JI, Chin JH, Kim WJ, Kim HR, et al. Impact of anesthetic agents on overall and recurrence-free survival in patients undergoing esophageal cancer surgery: A retrospective observational study. Scientific Reports. 2017;7(1):14020.

19. Oh TK, Kim K, Jheon S, Lee J, Do SH, Hwang JW, et al. Long-Term Oncologic Outcomes for Patients Undergoing Volatile Versus Intravenous Anesthesia for Non-Small Cell Lung Cancer Surgery: A Retrospective Propensity Matching Analysis. Cancer Control. 2018;25(1):1073274818775360.

20. Zheng $X$, Wang $Y$, Dong L, Zhao S, Wang L, Chen H, et al. Effects of propofol-based total intravenous anesthesia on gastric cancer: a retrospective study. OncoTargets and therapy. 2018;11:1141.

21. Wu ZF, Lee MS, Wong CS, Lu CH, Huang YS, Lin KT, et al. Propofol-based Total Intravenous Anesthesia Is Associated with Better Survival Than Desflurane Anesthesia in Colon Cancer Surgery. Anesthesiology. 2018;129(5): 932-941.

22. Soltanizadeh S, Degett T H, Gögenur I. Outcomes of cancer surgery after inhalational and intravenous anesthesia: A systematic review. Journal of clinical anesthesia. 2017;42:19-25.

23. Wells GA, Shea B, O'Connell D, Petersen J, Welch V, Losos M, et al. The Newcastle-Ottawa Scale (NOS) for assessing the quality of nonrandomized studies in meta-analyses. Department of Epidemiology and Community Medicine, University of Ottawa, Canada. www. ohr i. ca/programs/clinical_ epidemiology/oxford. htm.

24. Soltanizadeh S, Degett TH, Gögenur I. Outcomes of cancer surgery after inhalational and intravenous anesthesia: A systematic review. J Clin Anesth. 2017;42:19-25.

25. Yap A, Lopez-Olivo MA, Dubowitz J, Hiller J, Riedel B; Global Onco-Anesthesia Research Collaboration Group. Anesthetic technique and cancer outcomes: a meta-analysis of total intravenous versus volatile anesthesia. Can J Anaesth. 2019;66(5):546-561.

26. Wells GA, Shea B, Higgins JP, Sterne J, Tugwell P, Reeves BC. Checklists of methodological issues for review authors to consider when including nonrandomized studies in systematic reviews. Res Synth Meth. 2013;4:63e77.

27. Valentine JC, Thompson SG. Issues relating to confounding and meta-analysis when including non-randomized studies in systematic reviews on the effects of interventions. Res Synth Meth. 2013;4:26e35.

28. Reeves BC, Higgins JPT, Ramsay C, Shea B, Tugwell P, Wells GA. An introduction to methodological issues when including non-randomized studies in systematic reviews on the effects of interventions. Res Synth Meth. 2013;4:1e11. 
29. Myles PS, Peyton P, Silbert B, Hunt J, Rigg JR, Sessler DI. Perioperative epidural analgesia for major abdominal surgery for cancer and recurrence-free survival: randomised trial. Bmj. 2011;342:d1491.

30. Wunsch H, Linde-Zwirble WT, Angus DC. Methods to adjust for bias and confounding in critical care health services research involving observational data. J Crit Care. 2006;21:1e7.v

31. Bradburn MJ, Clark TG, Love SB, Altman DG. Survival analysis Part III: multivariate data analysis e choosing a model and assessing its adequacy and fit. Br J Cancer. 2003;89:605e11.

32. Parsons LS. Reducing Bias in a Propensity Score Matched-Pair Sample Using Greedy Matching Techniques [EB/OL]. Seattle, WA: Ovation Research Group. Available from: http://www2.sas.com/proceedings/sugi26/p214-26.pdf. Accessed September 22, 2010.

33. Rosenbaum P R, Rubin D B. Constructing a control group using multivariate matched sampling methods that incorporate the propensity score. The American Statistician. 1985;39(1): 33-38.

34. Woo JH, Baik HJ, Kim CH, Chung RK, Kim DY, Lee GY, et al. Effect of propofol and desflurane on immune cell populations in breast cancer patients: a randomized trial. Journal of Korean medical science. 2015;30(10):1503-1508.

35. Mitsuhata H, Shimizu R, Yokoyama MM. Suppressive effects of volatile anesthetics on cytokine release in human peripheral blood mononuclear cells. International journal of immunopharmacology. 1995;17(6):529-534.

36. Salo M, Pirttikangas C O, Pulkki K. Effects of propofol emulsion and thiopentone on T helper cell type-1/type-2 balance in vitro. Anaesthesia. 1997;52(4):341-344.

37. Christopherson R, James KE, Tableman M, Marshall P, Johnson FE. Long-term survival after colon cancer surgery: a variation associated with choice of anesthesia. Anesthesia \& Analgesia. 2008;107(1):325-332.

38. Coleman MP, Quaresma M, Berrino F, Lutz JM, De Angelis R, Capocaccia R, et al. Cancer survival in five continents: a worldwide population-based study (CONCORD). The lancet oncology. 2008;9(8):730-756.

39. Gupta G P, Massagué J. Cancer metastasis: building a framework. Cell. 2006;127(4):679-695.

40. Kaptchuk TJ. The double-blind, randomized, placebo-controlled trial: gold standard or golden calf? Journal of clinical epidemiology. 2001;54(6):541-549.

41. Higgins JP, Altman DG, Gøtzsche PC, Jüni P, Moher D, Oxman AD, et al. The Cochrane Collaboration's tool for assessing risk of bias in randomised trials. Bmj. 2011;343:d5928.

42. Udumyan R, Montgomery S, Fang F, Almroth H, Valdimarsdottir U, Ekbom A, et al. Beta-Blocker Drug Use and Survival among Patients with Pancreatic Adenocarcinoma. Cancer Res. 2017;77(13):3700-3707.

\section{Tables}

เble 1 Characteristics of included studies 


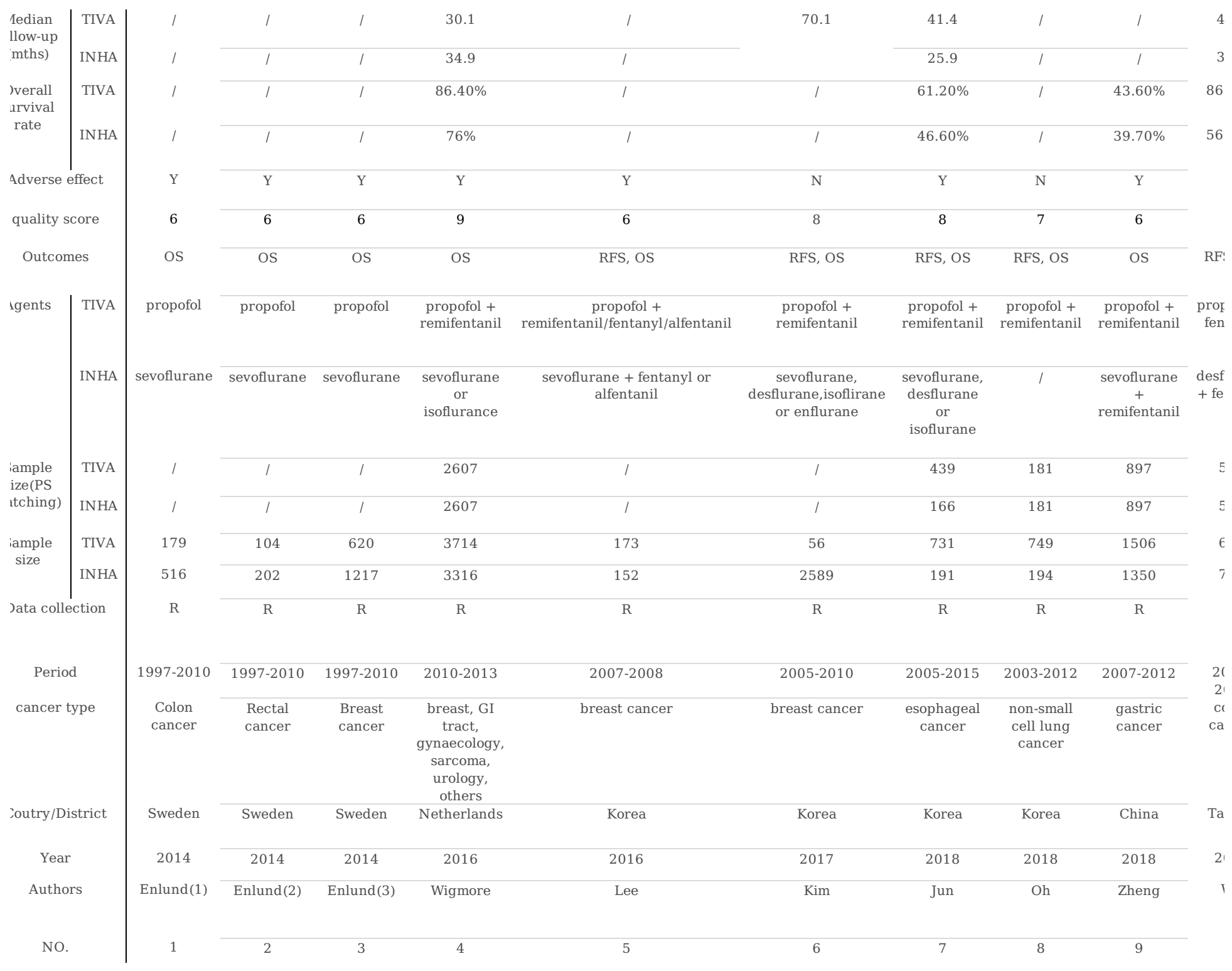

Table 2 Overall and recurrence free survival summary data 


\begin{tabular}{|c|c|c|c|c|c|c|}
\hline \multirow[t]{2}{*}{ tuthors } & \multicolumn{3}{|c|}{ OS HR (CI 95\%) } & \multicolumn{3}{|c|}{ RFS HR (CI 95\%) } \\
\hline & $\begin{array}{c}\text { Non- } \\
\text { Adjusted }\end{array}$ & $\begin{array}{c}\text { Adjusted by multivariate } \\
\text { analysis }\end{array}$ & $\begin{array}{l}\text { Adjusted by PS and } \\
\text { multivariate analysis }\end{array}$ & Non-Adjusted & $\begin{array}{c}\text { Adjusted by multivariate } \\
\text { analysis }\end{array}$ & $\begin{array}{l}\text { Adjusted by PS and } \\
\text { multivariate analysis }\end{array}$ \\
\hline nlund(1) & $\begin{array}{c}1.35(1.08- \\
1.67)\end{array}$ & $1.06(0.8-1.41)$ & $1.08(0.83-1.41)$ & 1 & l & 1 \\
\hline alund(2) & $\begin{array}{c}1.25(0.82- \\
1.92)\end{array}$ & $1.15(0.73-1.82)$ & $1.15(0.73-1.82)$ & 1 & l & l \\
\hline alund(3) & $\begin{array}{c}1.19(0.93- \\
1.54)\end{array}$ & $0.76(0.52-1.08)$ & $0.76(0.52-1.08)$ & 1 & l & l \\
\hline Vigmore & $\begin{array}{c}1.8(1.61- \\
2.02)\end{array}$ & $1.46(1.23-1.66)$ & $1.46(1.23-1.66)$ & 1 & 1 & I \\
\hline Lee & $\begin{array}{c}1.26(0.24- \\
6.52)\end{array}$ & 1 & 1 & $\begin{array}{c}1.818(1.028- \\
3.215)\end{array}$ & $2.092(1.16-3.774)$ & I \\
\hline Kim & 1 & $2.967(0.721-12.216)$ & $4.83(1-23.2)$ & $\begin{array}{c}0.857(0.38- \\
1.929)\end{array}$ & $1.136(0.496-2.597)$ & $1.28(0.59-2.78)$ \\
\hline Jun & $\begin{array}{c}1.5(1.19- \\
1.89)\end{array}$ & $1.58(1.24-2.01)$ & $1.57(1.19-2.07)$ & $\begin{array}{c}1.48(1.18- \\
1.86)\end{array}$ & $1.42(1.12-1.79)$ & $1.36(1.04-1.77)$ \\
\hline $\mathrm{Oh}$ & $\begin{array}{c}1.12(0.79- \\
1.6)\end{array}$ & $1.15(0.9-1.48)$ & $1.11(0.79-1.56)$ & $1.3(0.81-2.09)$ & $0.75(0.52-1.07)$ & $0.76(0.49-1.19)$ \\
\hline Zheng & $\begin{array}{c}1.59(1.43- \\
1.79)\end{array}$ & $1.64(1.45-1.82)$ & $1.54(1.33-1.79)$ & & 1 & 1 \\
\hline $\mathrm{Wu}$ & $\begin{array}{c}3.7(2.86- \\
4.557)\end{array}$ & $2.78(2.13-3.57)$ & l & $2.58(1.2-32)$ & l & I \\
\hline
\end{tabular}

\section{Figures}




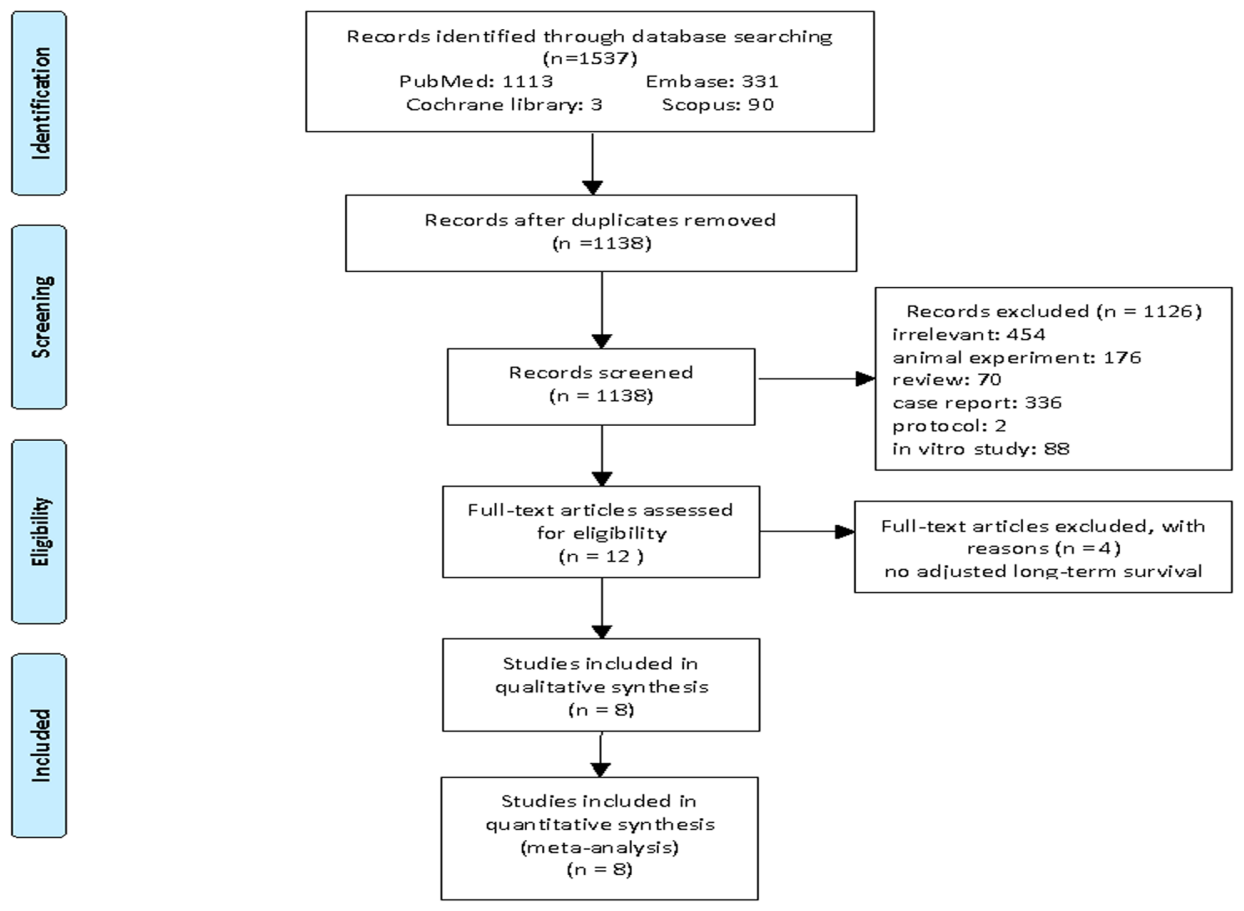

\section{Figure 1}

Study flow diagram: selection of studies for inclusion in the systematic review and meta-analysis. 


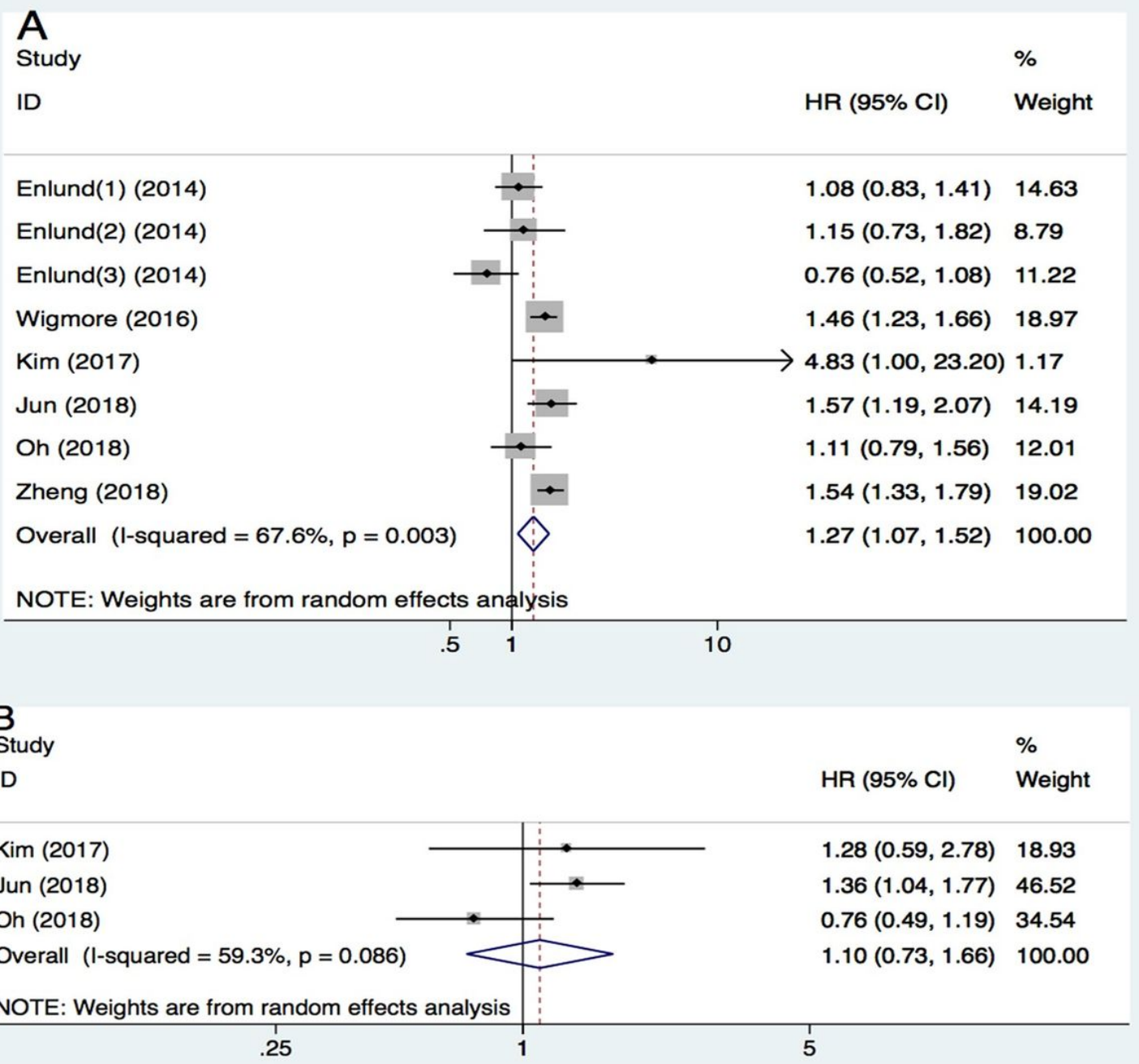

\section{Figure 2}

Forest plots and results of the meta-analysis of multivariate and PS adjusted studies: a) Hazard ratios (HRs) for OS by INHA vs. TIVA. b) HRs for RFS by INHA vs. TIVA. $95 \% \mathrm{Cl}$ : confidence interval at $95 \%$, W: weight. 
A

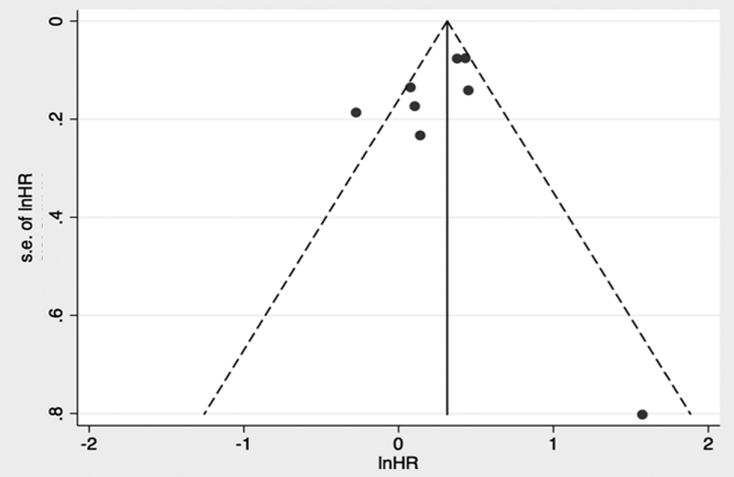

B

Study

ID

$\mathrm{HR}(95 \% \mathrm{Cl})$

OS

unadjusted

multivariate adjusted

multivariate and PS adjusted

RFS

unadjusted

multivariate adjusted

multivariate and PS adjusted

NOTE: Weights are from random effects analysis

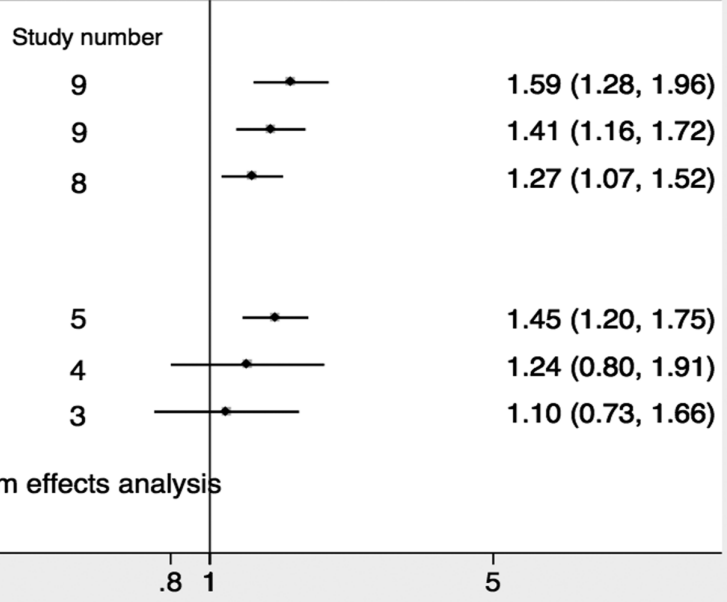

\section{Figure 3}

a) Funnel plot analysis to detect publication bias for OS studies. Each point represents an individual study for the indicated association. b) Graphical comparison among non-adjusted, multivariate adjusted, multivariate and PS adjusted methods for OS. HR: hazard ratio; MV: multivariable.

\section{Supplementary Files}

This is a list of supplementary files associated with this preprint. Click to download.

- PRISMANMAchecklist.doc 